\title{
Military Blast Injury and Chronic Neurodegeneration: Research Presentations from the 2015 International State-of-the-Science Meeting
}

\author{
*Denes Agoston,' Peethambaran Arun,2 Patrick Bellgowan, Steven Broglio, Robert Cantu, \\ David Cook, ${ }^{6}$ Uade Olaghere da Silva, Dara Dickstein, Gregory Elder, Elizabeth Fudge, ${ }^{10}$ \\ Sam Gandy,, 11 Jessica Gill, ${ }^{12}$ John F. Glenn, ${ }^{13}$ Raj K. Gupta, ${ }^{13}$ Sidney Hinds, ${ }^{14}$ Stuart Hoffman, ${ }^{15}$ \\ Theresa Lattimore, ${ }^{10}$ Alexander Lin, ${ }^{16}$ Kun Ping Lu, ${ }^{17}$ Joseph Maroon, ${ }^{18}$ David Okonkwo, ${ }^{18}$ \\ Daniel Perl, Meghan Robinson, ${ }^{19}$ Charles Rosen, ${ }^{20}$ and Douglas Smith ${ }^{21}$
}

\begin{abstract}
Blast-related traumatic brain injury (TBI) is a signature injury of recent military conflicts, leading to increased Department of Defense (DoD) interest in its potential long-term effects, such as chronic traumatic encephalopathy (CTE). The DoD Blast Injury Research Program Coordinating Office convened the 2015 International State-of-the-Science Meeting to discuss the existing evidence regarding a causal relationship between TBI and CTE. Over the course of the meeting, experts across government, academia, and the sports community presented cutting edge research on the unique pathological characteristics of blast-related TBI, blast-related neurodegenerative mechanisms, risk factors for CTE, potential biomarkers for CTE, and treatment strategies for chronic neurodegeneration. The current paper summarizes these presentations. Although many advances have been made to address these topics, more research is needed to establish the existence of links between the long-term effects of single or multiple blast-related TBI and CTE.
\end{abstract}

Keywords: blast injury; chronic traumatic encephalopathy; neurodegeneration; repetitive head trauma; traumatic brain injury

\footnotetext{
${ }^{1}$ Uniformed Services University of the Health Sciences, Bethesda, Maryland.

${ }^{2}$ Walter Reed Army Institute of Walter Reed Army Institute of Research, Silver Spring, Maryland.

${ }^{3}$ National Institute of Neurological Disorders and Stroke, Bethesda, Maryland.

${ }^{4}$ University of Michigan, Ann Arbor, Michigan.

${ }^{5}$ Boston University School of Medicine, Boston, Massachusetts.

${ }^{6}$ VA Puget Sound Health Care System, Seattle, Washington.

${ }^{7}$ Naval Medical Research Center, San Diego, California.

${ }^{8}$ Icahn School of Medicine at Mount Sinai, New York, New York.

${ }^{9}$ James J. Peters VA Medical Center, Bronx, New York.

${ }^{10}$ Office of the Assistant Secretary of Defense, Health Affairs, Falls Church, Virginia.

${ }^{11}$ James J. Peters VA Medical Center, Bronx, New York.

${ }^{12}$ National Institutes of Health, Bethesda, Maryland.

${ }^{13}$ US Army Medical Research and Materiel Command, Fort Detrik, Maryland.

${ }^{14}$ Defense and Veterans Brain Injury Center, Rockville, Maryland.

${ }^{15}$ Department of Veterans Affairs, Washington, DC.

${ }^{16}$ Brigham and Women's Hospital, Harvard Medical School, Boston, Massachusetts.

${ }^{17}$ Beth Israel Deaconess Medical Center, Harvard Medical School, Boston, Massachusetts.

${ }^{18}$ University of Pittsburgh Medical Center, Pittsburgh, Pennsylvania.

${ }^{19}$ VA Boston Healthcare System, Boston, Massachusetts.

${ }^{20}$ Department of Neurosurgery, West Virginia University, Morgantown, West Virginia.

${ }^{21}$ University of Pennsylvania, Philadelphia, Pennsylvania.

*Authors are listed alphabetically.
} 


\section{Introduction}

$\mathbf{E}$ XPOSURE TO BLAST is a common occupational hazard for service members, and blast-induced head injury and associated psychological trauma are frequently referred to as signature injuries of recent conflicts. Given its prevalence and relative newness as an injury, there is a particular interest in the long-term consequences of blast-related TBI, especially repeated trauma. Ensuring brain health to enhance and sustain force readiness across the military life cycle is therefore a major focus for the Department of Defense (DoD) (see Fig. 1). ${ }^{1}$ Modern military medical needs have changed in an era in which a majority of service members who sustain injury seek a return to duty. In addition, the military health system must be able to help persons stand down from duties when needed, recover as rapidly as possible, and return to full function.

Scientific and medical research informs DoD policy that seeks to mitigate the effects of blast-related traumatic brain injury (TBI) while maintaining operational priorities. The long-term effects of blast- and impact-related TBI are largely unknown, and there is growing concern that individuals with a history of head injury, with or without a diagnosis of TBI, may be at increased risk for chronic traumatic encephalopathy (CTE) developing. The potential for progressive neurodegenerative conditions like CTE raises critical questions about the identification and interventions for at-risk individuals.

At the 2015 International State-of-the-Science (SoS) Meeting held by the DoD Blast Injury Research Program Coordinating Office, participants sought to establish a shared understanding of the current scientific knowledge of blast-related trauma and the development of chronic neurodegeneration. During the first one and a half days of the meeting, speakers from government and academia summarized research community perspectives and research findings, setting the stage for the working group discussions and expert panel deliberations during the next two days (Outcomes from the working groups and expert panel are included in this Supplement). Keynote and topic presentations discussed service member needs, the prevalence of military and civilian TBI, policy considerations, government program efforts, and perspectives from the research community. These presentations set the stage for 15 subsequent cutting-edge research presentations, which served to inform the state of the science. This article summarizes these 15 research presentations.

\section{Setting the Stage}

More than $82 \%$ of DoD TBIs reported in the last 15 years were diagnosed as mild TBI (mTBI), ${ }^{2}$ which is also known as "concussion." More than $80 \%$ all TBIs are diagnosed in nondeployed environments. Blast-induced TBI is a common mechanism of injury; for example, vehicle blast was the cause for 39\% of TBIs in Operation Enduring Freedom (OEF) and $51 \%$ percent of TBIs in Operation Iraqi Freedom (OIF). The Blast Exposure and Concussion Incident Report identified 16,760 service members as having been involved in a potentially concussive event between August 2010 and June 2014; of those service members, 2734 (16.3\%) received a diagnosis of concussion.

The DoD conducts numerous studies aimed at identifying the results of blast-related head trauma such as TBI. Recently, the Naval Medical Research Center, in collaboration with the Walter Reed Army Institute of Research Blast Research Program, studied mortar and artillery operators exposed to blast pressures during training. ${ }^{3}$ Both positions report symptoms of persistent headache, mood disorders, and sleep disturbances, particularly among more experienced operators and instructors. Mean and peak overpressure exposure was studied in mortar and artillery operators using helmet-mounted sensors. For both types of weaponry, mean and

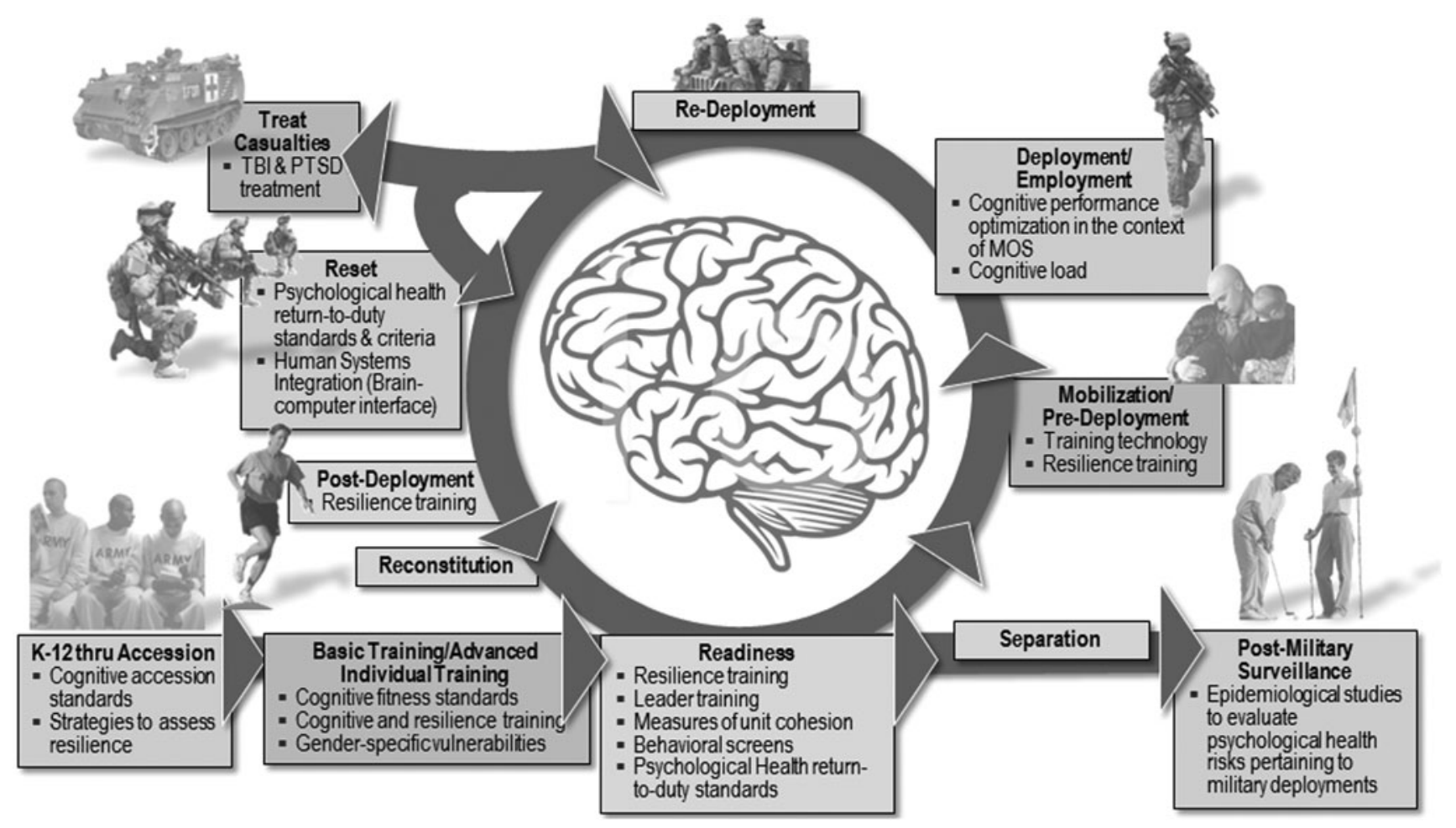

FIG. 1. Brain health across the military life cycle. 
peak overpressure varied by operator role (i.e., position relative to weapon when carrying out role) and direction from operator. Peak overpressure was highest for the chief of section role, while mean overpressure was generally consistent across roles. Artillery radio telephone operators, who are generally at a greater relative distance from weaponry, were exposed to unexpected levels of mean overpressure, which may have been the result of blast waves rebounding off nearby surfaces and vehicles.

The acute and chronic effects of TBI are also being studied by the Readiness Division of the Healthcare Operations Directorate at the Defense Health Agency (DHA), which collaborates with external partners to identify actions for the prevention and management of TBI that could be codified to make a beneficial impact across the DoD (see Fig. 2). ${ }^{4}$ To implement these actions, Operational Medicine supports Health Affairs (HA) for policy-level initiatives (e.g., action memoranda, DoDIs) or DHA for logistical or implementation-level initiatives.

Current TBI-related policy initiatives include the HA TBI definition and reporting memo, DoDI 6490.11 (requiring line leaders to report potentially concussive events), DoDI 6490.13 (requiring preand post-deployment neurocognitive assessments), service-specific policies, and combatant command policies. Aspects of these policy initiatives seek to codify and standardize TBI reporting, in part to enable future service member and veteran electronic health records to track and identify at-risk beneficiaries as clinical understanding of the interaction between TBI exposure and chronic health outcomes advances. Recent demonstrations of significant gaps in point-of-injury documentation and data collection highlight policy challenges that need to be addressed to ensure that future health records are able to identify at-risk individuals in the health system.

The DoD also works in partnership with other federal government organizations to promote brain health research. Veterans are a key population for medical and research efforts, and the Veterans Affairs (VA) has many TBI research efforts dedicated to improving the future of care for veterans with brain injury. There are more veterans who have survived blast injuries and TBI than ever before, and the VA's mission is to understand the nature of brain injuries and ultimately reintegrate veterans back into their communities.

To that end, and in response to Executive Order 13625 (Section 5: PTSD, TBI, Suicide Prevention), the VA worked with the DoD, the Department of Health and Human Services and the Department of Education to establish the National Research Action Plan (NRAP), which coordinates federal activities across the continuum-from research to clinical care practice. ${ }^{5}$ Stemming from the NRAP, the Chronic Effects of Neurotrauma Consortium (CENC) develops projects across several participating national research sites dedicated to exploring the long-term effects of TBI. The CENC is charged with examining the association between chronic TBI and common comorbidities; determining whether a causal link exists between mTBI and neurodegenerative disease and other comorbidities; identifying diagnostic and predictive indicators of neurodegenerative disease; and developing/advancing methods to treat those with chronic neurodegenerative disease to improve the health of veterans.

The most recent VA State of the Art Conference established a number of recommendations for future TBI research, including understanding the effects of gender differences in TBI outcomes; developing standardized animal injury models using common data elements; examining genetic markers for injury responses, recovery, and resilience; distinguishing the effects of injury type and number of insults on the mechanisms of degeneration; and improving the reach, efficacy, and acceptance of telemedicine for rehabilitation and clinical monitoring. These recommendations reflect the VA's policy commitment to determining and addressing the long-term effects of repeated exposures of blast and blunt trauma on the brain.

Government-supported research on brain health topics, such as the pathophysiology of CTE, is conducted by the National Institute

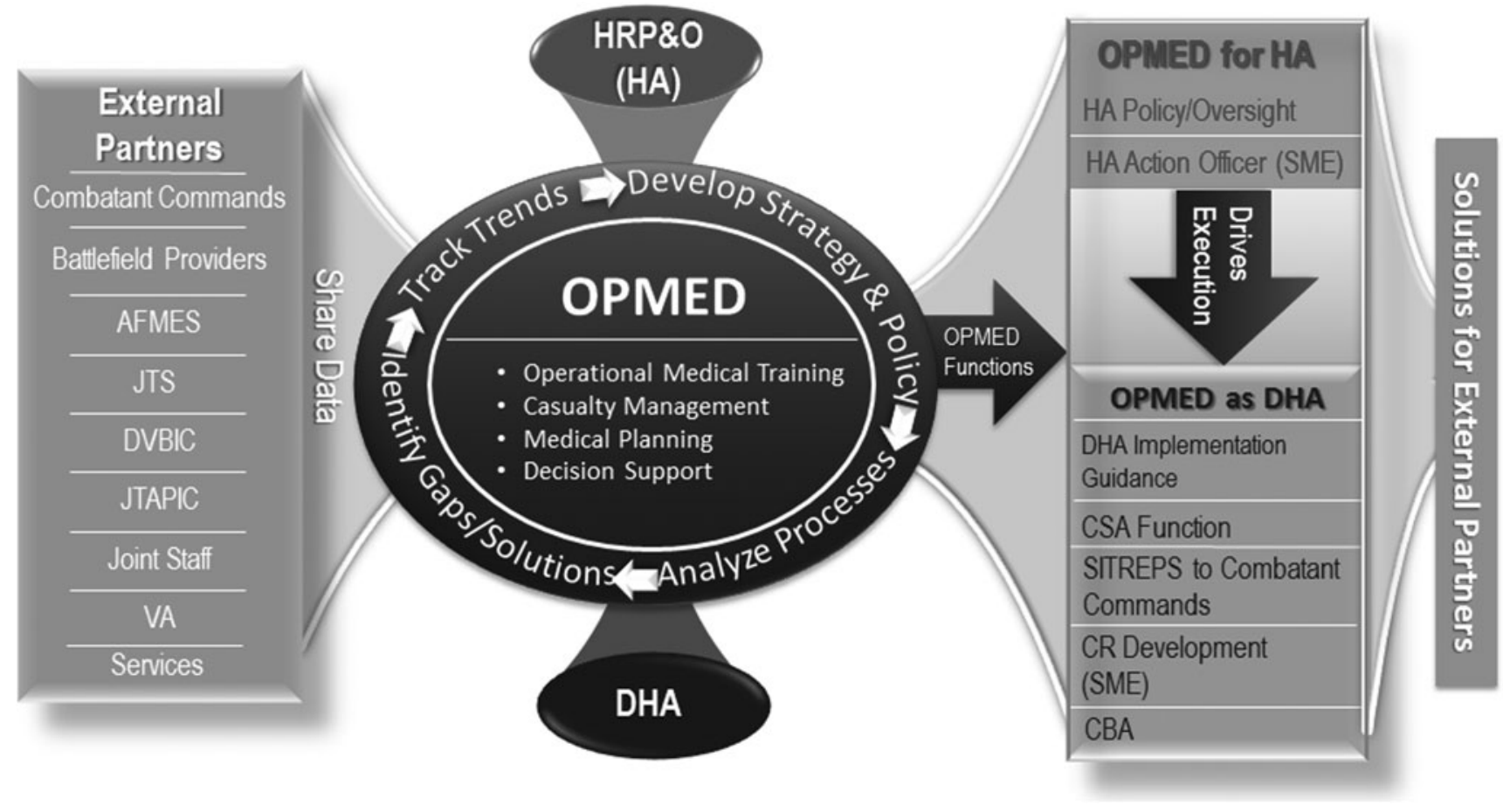

FIG. 2. Readiness division. 
of Neurological Disorders and Stroke (NINDS). The NINDS is one of 12 National Institutes of Health (NIH) supporting TBI research, which, across intramural and extramural initiatives, addresses basic science and clinical investigations. Since 2013, the NINDS has been funding post-mortem CTE studies to address gaps in understanding, including neuropathological characteristics, incidence, and prevalence. In 2015, the NINDS supported a consensus workshop with the goal of defining required criteria for neuropathological diagnosis of CTE. ${ }^{6}$ Attendees established consensus about the post-mortem neuropathological features required for CTE and identified features supporting a neuropathological diagnosis of CTE.

The NINDS is preparing to release funding for ante-mortem research projects focusing on brain imaging and other biomarkers for detection, measurement, and characterization of CTE and its progression over a three- to five-year period in persons with a possible CTE diagnosis. ${ }^{6}$ The goal is to form a better understanding of the neurological mechanisms that could lead to a consensus diagnosis of CTE and that could be used in future clinical trials aimed at preventing or slowing disease progression. The NIH-funded CTE studies will collect, and bank, a variety of biomarkers and genetic data. Data from these studies will use the common data elements from the Federal Interagency Traumatic Brain Injury Research (FITBIR) data dictionary and will be made available for secondary use in FITBIR, thereby maximizing the NINDS's research investment and enabling more rapid solutions using a big data approach.

Another federal program advancing CTE research is the Metabolomics Workbench, which gives NIH-funded investigators supplementary funds for data analysis into the unique chemical fingerprints that specific cellular processes leave behind. ${ }^{6}$ The Enhancing NeuroImaging Genetics through Meta-Analysis (ENIGMA) Consortium combines imaging and genetic data from datasets across the world. Currently, there is no TBI-specific effort within ENIGMA, but the aim is to develop a working group that will enable the concatenation of TBI datasets.

Research involving head trauma in athletes may also shed light on these conditions, ${ }^{7}$ particularly given that $75-80 \%$ of military concussions are unrelated to combat. ${ }^{8}$ Research studies of retired professional football players with three or more reported concussions found a higher prevalence of mild cognitive impairment and memory problems when compared with retirees without a history of concussion. ${ }^{9}$ Later studies of college-age students with a self-reported history of concussion, however, revealed no difference in cognitive performance compared with those without a history of concussion. ${ }^{10,11}$

Differences in these findings may be explained by differences between study populations or that cognitive measures used in the collegiate population were not sensitive enough to detect changes. Subjects with a history of concussion exhibited significantly different electrophysiological response compared with those without concussion, as measured by event-related potentials during sensory processing behavioral tasks ${ }^{11}$; these electrophysiological observations are thought to reflect subtle changes potentially in the brain resulting from concussion.

A subsequent study of functional balance and gait found that former high school athletes with a history of concussion had significantly different balance patterns and gait mechanics compared with those without concussion. ${ }^{12,13}$ That these balance and gait behaviors are also seen in aging led to the proposal of an accelerated decline hypothesis - that, because of exposure to head injury and other potential factors, concussed persons have a more rapid decline in performance as they age compared with those without concussion. ${ }^{14}$ Recent unpublished work, however, indicates that declines across age in cognitive function and gait do not differ between concussed and nonconcussed former high school athletes, raising questions about the accelerated decline hypothesis. Additional studies examining concussion in those presumed to have more extensive exposure, such as college athletes and professional athletes, are needed to further explore the accelerated decline hypothesis.

The NCAA and DoD are collaborating on the Grand Alliance Concussion, Assessment, Research and Education Consortium, a three-year prospective study across 30 colleges and universities to monitor the natural history of concussion in collegiate athletes and cadets at military academies 20 to 40 years post-injury. To date, 14,000 athletes and cadets have been enrolled, and 398 have received a concussion diagnosis. This study will be the most comprehensive study of concussion and head impact ever conducted and may provide critical insight to the risks and management of concussion and treatment options for those with concussion.

Little is known about the possible long-term structural, emotional, and psychological effects of concussion in military and sports. ${ }^{15}$ This lack of knowledge has prompted research into understanding the pathological mechanisms of TBI and other clinical factors associated with CTE. Experts have observed that athletes generally recover more quickly from concussion than soldiers; the reasons for the observation are not clear, but some believe the unique aspects of blast injury are responsible for the differences in recovery time.

A recent two-day conference at the University of Pittsburgh concluded that the benefits of oft-prescribed complete rest after concussion may be limited or detrimental and that early physical activity, in some cases, may be superior to promote neurogenesis, synaptogenesis, and neuroplasticity. ${ }^{15}$ In the last decade, leading clinical neuropathologists have been examining the brains of athletes and military service members suspected of having CTErelated neurocognitive changes after a history of TBI or blast injury to the brain. While researchers have explored the potential risk factors of CTE, based mainly on case reports, the only consistent risk factor supported by current evidence is a previous exposure to concussion or TBI.

Researchers are also investigating multiple biological mechanisms potentially unifying the underlying pathogenesis of concussion, post-traumatic stress disorder (PTSD), and CTE. Concussion is known to be caused initially by an acute neurometabolic mismatch of neuronal glucose, potassium, and calcium released immediately after brain trauma. ${ }^{16,17}$ Another proposed immunoexcitotoxic mechanism posits that repeated TBI, without intervening brain healing, may induce brain-derived microglia to release destructive inflammatory cytokines, chemokines, and proteases continuously ${ }^{18}$; in addition, the release of excitotoxic neurotransmitters (glutamate) might lead to the neurodegenerative changes seen in CTE. Multiple frequent concussions may activate microglia chronically, which accelerates the inflammatory and excitotoxic events and interrupts the reparative mechanisms (e.g., growth factor release) that normally occur after a single injury to heal the brain. Studies have also suggested that activation of microglia can occur without exposure to physical brain trauma in mental health disorders such as schizophrenia ${ }^{19}$ and PTSD. $^{20}$

\section{Research Presentations}

1. "Why Do We Know So Little about the Neuropathology of Blast TBI?"' by Professor Douglas Smith (presented by Dr. William Stewart) 
2. "Neuropathology Studies of Acute and Chronic Blast TBI, Evidence of an Unique Pattern of Damage to the Human Brain Following Blast Exposure," by Dr. Daniel Perl

3. "Clinicopathologic Phenotypes of Chronic Traumatic Encephalopathy: Distinct Risk Factors," by Dr. Robert Cantu

4. "Vascular and Inflammatory Factors in the Chronic Pathophysiology of Blast-Induced Brain Injury," by Dr. Gregory Elder

5. "Repeated Blast Exposures Cause Neuropathological Underpinnings of CTE and Alzheimer's Disease," by Dr. Peethambaran Arun

6. "Measuring Changes in Brain Chemistry after Repetitive Brain Trauma Using MR Spectroscopy,"' by Dr. Alexander Lin

7. "Chronic Traumatic Encephalopathy: Contact Sports and Blast Injuries," by Dr. Joseph Maroon

8. "The Role of Aberrant Phospho-Tau in Blast-Induced Mild Traumatic Brain Injury," by Dr. David Cook

9. "A Novel Investigation into the Underlying Mechanisms Linking Neurotrauma to Neurodegeneration," by Dr. Charles Rosen

10. "High-Fidelity Animal Modeling of Blast-Induced Neurodegeneration: A Longitudinal Study to Identify Mechanisms and Biomarkers," by Prof. Denes Agoston

11. "Neuroimaging of Nonconcussive Blast Exposure in $\mathrm{OEF} /$ OIF/OND Veterans," by Dr. Meghan Robinson

12. "Blast TBIs Are Associated with Higher Concentrations of Amyloid Beta in Peripheral Blood," by Dr. Jessica Gill

13. "PET Imaging with Pittsburgh Compound B of Amyloid Deposition in White Matter in Chronic TBI: An Exploratory Analysis," by Dr. David Okonkwo

14. "Cortical Gray Matter-White Junction Pattern of Retention of the Tauopathy Ligand 18F-T807 (Avid 1451) in Clinically Probable CTE," by Prof. Sam Gandy (presented by Dr. Dara Dickstein)

15. "Cis-P-Tau Directly Links TBI to CTE and AD, but Can Be Blocked by Antibody," by Dr. Kun Ping Lu

\section{Pathological characteristics of blast-induced TBI}

Researchers use neuropathological evidence to identify and characterize CTE. These neuropathological observations are also a window into the mechanisms that may drive chronic neurodegeneration after TBI.

Dr. William Stewart, Queen Elizabeth University Hospital, presented on behalf of Dr. Douglas Smith from the University of Pennsylvania on lessons learned from studies in animal models regarding the neuropathology of blast-induced TBI. Diffuse axonal injury (DAI) is generally believed to be the primary clinical neuropathological sequela in impact (nonblast) mTBI or concussion. Pre-clinical studies in blast, however, have failed repeatedly to demonstrate DAI acutely in primate, ${ }^{21}$ swine $^{22}$ and rodent ${ }^{23,24}$ models. Evidence in support of axonal injury is also an inconsistent finding in imaging and pathology studies in humans; potential confounding factors where axonal pathology has been described at autopsy include drug overdose, ante-mortem anoxia, and concurrent impact $\mathrm{TBI}^{25}$

While DAI is not a consistent observation in pure blast TBI models, reports across species have documented evidence of glial, $^{21}$ microglial, ${ }^{26}$ ) and vascular pathologies, however. ${ }^{22}$

Better neuropathological characterization of blast-induced pathology in human tissue is a high-priority research need that will inform and direct the development of appropriate animal models. In addition, ongoing efforts to define common data elements in preclinical research will help drive consensus between groups. Greater collaboration in the field is needed.

Opportunities for clinical neuropathological research are limited. Dr. Daniel Perl, of the Uniformed Services University of the Health Sciences (USUHS), reviewed neuropathology findings from case studies of acute and chronic blast TBI in humans, including observations of unique pathological lesions in the form of glial scarring.

Few neuropathological studies exist describing acute or chronic effects of blast TBI in humans. Observations of unusual glial scarring in an index case of chronic blast TBI, however, led to a recent collaboration between the USUHS Center for Neuroscience and Regenerative Medicine and the Joint Pathology Center comparing neuropathology after chronic blast TBI (five cases), acute/ subacute blast TBI (three cases), chronic civilian impact (nonblast) TBI (five cases), and civilian controls with no TBI history (three cases). A notable pattern of glial scarring was observed in all chronic blast TBI cases. A parallel pattern was seen in two service members who survived four days post-blast: reactive astrocytes (which are thought to reflect the initiation of glial scarring) were found in the subpial region and gray-white matter junction.

Ex vivo high-resolution magnetic resonance imaging (MRI) (7 Tesla) of tissue from a single chronic blast TBI case provided a preliminary indication that the detection of gray-white matter abnormalities associated with glial scarring may be possible in living persons. Glial scarring was observed in structural areas associated with known functions thought to be adversely affected by chronic TBI or CTE. The current theory regarding how blast waves interact with human physiology predicts that damage will occur at structures consisting of, or proximate to, tissues of different densities, which is the case of structures with glial scarring in this preliminary study. Tau pathology was not observed consistently across chronic blast TBI cases; however, it is possible that tau aggregation would have developed in these patients over time. Observed patterns of axonal pathology also did not differentiate between acute/subacute and chronic blast cases.

These preliminary findings were observed in spite of small sample size, limited data on blast exposure number and nature of injuries, limited post-concussive symptomology data, and limited exposure histories for nonblast (impact) TBI cases. Larger, controlled studies comparing blast and civilian impact TBI and using available clinical and operational data to inform exposure history are recommended. High-resolution imaging of lesions associated with gliosis may also benefit clinical studies of blast-exposed service members. Based on these preliminary observations, the development of animal models with human tau or that characterize post-blast gliosis would also be beneficial.

\section{Risk factors and CTE}

Dr. Robert Cantu and Mr. Philip Montenigro, both from Boston University Alzheimer's Disease and CTE Center, reviewed research on the neuropathological and clinical features of CTE. CTE is defined as a progressive neurodegenerative disorder in those with a history of brain trauma (frequently with multiple exposures) that results in cognitive, behavioral, mood, and/or motor deficits. In post-mortem analysis, CTE is distinguished from Alzheimer disease (AD) by its characteristic pattern of tauopathy, including perivascular accumulation of neurofibrillary tangles at sulcal depths and superficial cortical layers. ${ }^{27} \mathrm{~A}$ recent case series of 
neuropathologically confirmed CTE identified the presence of amyloid-beta $(\mathrm{A} \beta)$ deposition in $52 \%$ of CTE subjects. ${ }^{28}$ The presence of $\mathrm{A} \beta$ pathology in these subjects occurred at an earlier age with altered dynamics compared with the normal aging population and those with $\mathrm{AD}$. In addition, $\mathrm{A} \beta$ deposition was associated with a greater severity of CTE tauopathy and dementia symptoms. With support from the NINDS and National Institute of Biomedical Imaging and Bioengineering, consensus neuropathological diagnostic criteria for CTE were established recently. ${ }^{29}$

Currently, CTE can be diagnosed only by a post-mortem neuropathological examination. In an effort to identify a common clinical presentation of $\mathrm{CTE},{ }^{30}$ investigators performed a retrospective analysis of clinical histories, medical records, and next-ofkin reports among autopsy-confirmed CTE cases without comorbidities. In more than $70 \%$ of cases, symptoms occurred in three clinical domains: behavior, mood, and cognition. Symptom clusters gave rise to two subtypes: (1) behavioral/mood, which had an earlier age of onset and predominant symptoms of explosivity, rage, depression, with mild cognitive symptoms later in life; and (2) cognitive, which had a later age of onset and predominately memory, executive, and attention issues.

Recently, Cantu and Montenigro and associates proposed clinical criteria for the diagnosis of $\mathrm{CTE}^{31}$ and for the first time applied these criteria to two case reports. ${ }^{32}$ To date, studies to validate and improve on the proposed clinical criteria are ongoing; an expert clinical consensus panel has already utilized the proposed criteria to diagnose clinically 99 cases retrospectively while panel members remain blinded to the neuropathological diagnosis. $^{33}$

Brain injury is the primary known risk factor for CTE. For professional football players, the number of years played and years since retirement both correlate with CTE severity. ${ }^{27}$ Career length may be associated with CTE severity by serving as a proxy for the number of injuries accumulated; years since retirement may be associated with the amount of time neuropathology was allowed to progress. Next of kin reported concussion frequency and steroid use are not correlated with severity of CTE pathology. It is not yet known which specific aspect(s) of brain injury (source, frequency, location) influence the risk for or natural progression of CTE. Closed-head impacts in collision sports (e.g., football, boxing) cause sudden acceleration or deceleration of the brain inside the skull that may introduce internal brain injuries without visible fracture or contusion.

These closed-head brain injuries may result from forces propagated through two biomechanical pathways. The first is the "common" pathway to injury, which is homogeneous in nature and occurs where the brain is structurally vulnerable to injury. When strains overwhelm the underlying brain tissue through the common pathway, a previously described acute closed-head brain injury pattern occurs termed the "gliding contusion." 34 The chronic neuropathological characteristics of CTE share certain similarities to, and may be associated with, the acute closed-head brain injury pattern of the gliding contusion. The second aspect of a closed-head brain injury is the "focal" or "local" pathway, which is heterogeneous in nature and specific to the type and location of impact. Thus, closed-head brain injuries result from a combination of both common (homogeneous) and local (heterogeneous) pathways to tissue injury.

The common pathway is thought to play the major role in the accumulation of injury that results in long-term impairment and CTE tauopathy, as game play results in repetitive closed-head impacts. Cantu and Montenigro hypothesized, however, that sport specific differences in impact exposure might alter the pathological and clinical manifestations of CTE through differences in the biomechanical forces introduced through the local pathway of closed-head impacts.

Football players are exposed generally to repeated linear forces because of frontal, head-to-head contact. Boxers are exposed generally to rotational acceleration resulting from the hook-punch to the chin. Rotational acceleration is thought to have a greater effect on the mid-brain and cerebellum, which was observed in a recent case comparison of a single professional football player and single professional boxer. ${ }^{32}$ Tissue analysis from both individuals met consensus based neuropathological criteria for stage III CTE. Yet the boxer sustained greater neuronal dropout and tau-positive neurons within the dentate of the cerebellum. These neuropathological differences were mirrored in a secondary analysis of the data from McKee and colleagues ${ }^{27}$ : when professional boxers and football players were compared, boxers were more likely than football players to have CTE with motor symptoms and neurofibrillary tangles in the cerebellar dentate, a higher proportion of which were severe. ${ }^{32}$

\section{Blast-induced neurodegenerative mechanisms}

Dr. Gregory Elder, of the James J. Peters VA Medical Center, reviewed animal model research of blast-induced behavioral alterations and pathology. Investigators at the Naval Medical Research Center developed an animal model designed to mimic blastinduced mTBI. Exposure to multiple blasts resulted in no major neuropathological abnormalities, but was associated with transiently impaired spatial learning as assessed by the Morris Water Maze test. ${ }^{35}$

Distinguishing the overlapping symptoms of PTSD and postconcussion syndrome has proven difficult for clinicians and has created the need for animal models to study and tease apart the behavioral components of these conditions. Recent experiments have demonstrated the induction of PTSD-related traits after exposure to blast. In behavioral assessments, animals exposed to multiple blasts exhibited increased anxiety, acoustic startle, and cued fear responses when compared with controls. In blast-exposed animals, abnormal behavioral responses persisted for three days after exposure to a predator scent. These behavioral abnormalities were also accompanied by changes in stathmin 1 , a microtubuleassociated protein highly expressed in the amygdala that plays a role in learning and fear responses. Subsequent DNA methylation profiling in these animals has indicated epigenetic changes potentially underlying long-term, chronic effects. ${ }^{36}$

An increase of $\mathrm{A} \beta$ plaques has been reported after closed-impact TBI in humans ${ }^{37}$ and in animal models. ${ }^{38}$ Recent work in blastexposed rodents, however, indicates a decrease of $A \beta$ in rats at $24 \mathrm{~h}$ and one week post-blast. ${ }^{39}$ In addition, amyloid precursor protein (APP) levels increased in blast-exposed animal models, similar to observations after closed-impact TBI. To date, tau pathology or neurofibrillary tangles have not been observed in this blast-exposed animal model.

Blast-induced vascular pathology may be relevant to the study of CTE given that perivascular tau accumulation is one defining characteristic of the disorder. Exposure to multiple blasts is associated with vascular abnormalities, with dysmorphic vessels exhibiting irregular vessel walls, and debris in the lumen; the neurophil surrounding such vessels was normal, suggesting that blast effects are specific to the vasculature. Acute blast exposure in this animal model was also associated with a degradation of the 
glycocalix, a supportive matrix lining the inner walls of blood vessels, and poorer spatial learning performance; both abnormalities could be rescued with prophylactic application of hyaluronidase during blast exposure. Chronic effects of blast exposure include remodeling of the extracellular matrix in rats. In addition to chronic vascular effects, neuroinflammatory-related changes after blast exposure are being considered as a potential mechanism of injury. ${ }^{40}$

Dr. Peethambaran Arun, of the Center for Military Psychiatry and Neuroscience at the Walter Reed Army Research Institute, presented findings from two animal models of brain injury describing effects on phosphorylated tau (p-tau) and APP levels in the brain. Rats given a single exposure to blast injury (blast tube, $19 \mathrm{psi}$ ) or impact-acceleration injury (weight drop, $500 \mathrm{~g}$ from $250 \mathrm{~cm}$ above the skull's surface) showed decreased levels of tissue nonspecific alkaline phosphatase (TNAP) and elevated levels of ptau at 6 and $24 \mathrm{~h}$ in the brainstem, hippocampus, and cortex compared with a sham control group. Plasma levels of alkaline phosphatase enzyme activity were also reduced at 6 and $24 \mathrm{~h}$ after exposure to an impact injury, but not blast; this result may be explained by alkaline phosphatase coming into the plasma from internal organs after blast exposure. Levels of alkaline phosphatase in the brain decreased after both blast and impact injuries. At $24 \mathrm{~h}$, APP levels were elevated in the brainstem, hippocampus, and cortex of both injury groups. These data suggest two processes are set in motion by blast or impact injury: (1) a decrease in TNAP, which may lead to the accumulation of p-tau, and eventual development of CTE; and (2) an accumulation of APP, which may trigger an AD-like pathology.

Six hours after rats are repeatedly exposed to blast (two $19 \mathrm{psi}$ blasts within $1 \mathrm{~min}$ ), p-tau levels are elevated in many brain areas (e.g., brainstem, cortex, cerebellum, midbrain), and remain significantly elevated in the hippocampus at $24 \mathrm{~h}$ and 14 days. In rats exposed to two blasts, p-tau levels are compared with rats exposed to a single blast. The APP levels, while also higher after exposure to two blasts, did not increase at the 6-h time point, but were elevated at $24 \mathrm{~h}$ and remained so through the 14-day time point.

The lack of a common pattern of elevation for p-tau and APP supports the hypothesis that the neurobiological mechanism of CTE may be distinct from AD, although the accumulation of APP at later time points suggests that blast can trigger an AD-like pathology. Future neuropathological work will examine the pattern of p-tau deposits to determine whether it is located in the superficial layer of the cortex, as in CTE, or in the global layer, as is seen in $\mathrm{AD}$.

Dr. Alexander Lin, of the Brigham and Women's Hospital, reviewed research using magnetic resonance spectroscopy (MRS) to identify post-concussion neurochemical alterations that may serve as biomarkers for CTE. The MRS measures relative concentrations of brain metabolites in living persons and can characterize acute (minutes to days) and chronic (years) neurometabolic changes after injury that may be associated with CTE.

Early application of MRS demonstrated a potential role for predicting functional outcomes after severe head injury. Studies in comatose patients with severe head injury resulting from car accidents found that those with a less than $20 \%$ reduction of $\mathrm{N}$ acetylaspartate (NAA; a chemical found within neural cell bodies that is thought to correlate with the number of functioning neurons) had positive recovery outcomes; patients with a larger than $50 \%$ reduction in NAA remained in a persistent vegetative state. ${ }^{41}$ The MRS measurements later were shown to be predictive of Glasgow Coma Scores in adults with severe head injury. ${ }^{42}$
MRS can also characterize metabolic changes after mild brain injury. In a study of subjects with a single concussion, compared with controls, MRS showed an acute reduction of NAA that recovered over time. In subjects with two concussions, NAA reduction was similar but recovered more slowly. ${ }^{43}$

Research in athletes exposed to head trauma aims to identify metabolic changes that may serve as CTE biomarkers. The MRS of retired football players with CTE-related complaints and a history of multiple concussions indicated elevated levels of choline (a marker of cell damage) and lower levels of glutathione (a marker for neural formation) in the anterior cingulate compared with controls. ${ }^{44}$ Elevated levels of choline and myo-inositol (a potential marker of glial cells) were observed in soccer players compared with a control group of noncontact sport athletes. ${ }^{45}$

Development of two-dimensional (2D) MRS has enabled identification and quantification of previously undetectable brain metabolites. Application of 2D MRS in retired football players with CTE-related complaints and a history of multiple concussions indicated elevated levels of glutamate; however, the degree of glutamate did not correlate with the degree of concussion exposure. These and other observations have led researchers to hypothesize about changes in brain metabolite levels during exposure to injury and development of CTE. For example, a better understanding of the timing of glutamate decrease could help identify time points for the potential application of intervention strategies that mitigate its neurotoxic effects. Future research combining MRS with neuroimaging modalities may advance understanding of how neurometabolic changes after brain injury or CTE are localized.

Dr. David Cook, of the VA Puget Sound Health Care System and University of Washington, reviewed research findings describing alterations in p-tau expression, blood-brain barrier dysfunction, and cerebellar damage in the brain after blast injury. To investigate these phenomena, an animal model of blast injury was developed that uses a shock tube to generate a blast wave similar to trinitrotoluene in the open field. Rodents exposed to this blast wave exhibited aberrant p-tau in the hippocampus for up to 30 days after injury and transient perivascular p-tau accumulation was detected before abating within $4 \mathrm{~h}$ after exposure.

In vivo two-photon laser scanning microscopy was used to study blood-brain barrier integrity in blast-exposed animals. Transient microvascular alterations and microglial responses were observed in focal areas after blast exposure. Delayed blood vessel dysfunction and microglial/macrophage accumulation was observed, indicating that some instances of these phenomena are not directly related to the immediate biomechanics of blast exposure.

After blast exposure, the cerebellum appears to be susceptible particularly to blood-brain barrier disruption, especially in ventral areas, and cerebellar Purkinje cells exhibit p-tau accumulation and plasma membrane integrity alterations. In addition, the loss of Purkinje cells was observed after exposure to a single blast; cell loss was greater after exposure to multiple blasts. Purkinje cell losses are observed in patch-like microdomains. These observations are similar to reports of Purkinje cell loss in post-mortem neuropathological examination of former boxers. ${ }^{46}$ In animal models, blast exposure causes alterations in a motor task (rotarod) and elevated astrogliosis 30 days after injury. Similarly, veterans exposed to blast exhibit hypometabolism in the cerebellum that correlates with the number of exposures and sensorimotor integration symptoms.

Dr. Charles Rosen, Department of Neurosurgery at West Virginia University, discussed research investigating the role of endoplasmic reticulum (ER) stress responses in blast-induced neurodegeneration. The ER stress responses are thought to be associated with 
neurodegeneration through the induction of p-tau pathology. ${ }^{47-49}$ The ER stress response is mediated in part by two intracellular cascades: (1) the PRKR-like ER kinase (PERK) pathway, and (2) the inositol-requiring enzyme 1 pathway. Stressors to the ER disrupt normal equilibrium between these two pathways and induce relative overactivation of the PERK pathway, which elevates the transcription factor $\mathrm{C} / \mathrm{enhancer}$ binder protein homologous protein (CHOP), leading to cell apoptosis through activation of caspase proteins.

To investigate intracellular signaling pathways associated with ER stress response and blast exposure, a rodent model was developed using a blast tube apparatus. Validation experiments demonstrated induction of a physiologically relevant blast wave, and animals exposed to the blast wave exhibited astrogliosis, p-tau pathology, and activation of the PERK pathway with elevation of CHOP. Exposure to single or repeated blast induced maladaptive behavior (reduced anxiety), as assessed by the Elevated Plus Maze, despite retention of normal motor activity. In addition, these animals exhibited delayed learning and memory deficits 21 days after blast, as assessed by the Morris Water Maze. Administration of salburinal $30 \mathrm{~min}$ before blast exposure inhibited activation of the PERK cascade and restored normal behavior on the Elevated Plus Maze. These data indicate that blast exposure induces ER stress responses that mediate TBI-relevant behavioral outcomes. Modification of the ER stress response could prove to be a beneficial strategy for preventing blast-related neurodegeneration and abnormal behavior. Complete details on the study by Dr. Rosen and colleagues can be found in the article "Endoplasmic Reticulum Stress Modulation as a Target for Ameliorating Effects of Blast Induced Traumatic Brain Injury" in this Supplement on page S-62.

\section{Neuroimaging and biomarkers}

The successful development of objective biomarkers could enable the identification of CTE pathology in living persons, which would greatly enhance research capabilities and would inform potential diagnostic and treatment strategies. Investigators are pursuing neuroimaging modalities and biospecimen analysis as potential biomarkers of CTE.

Dr. Denes Agoston, from USUHS, presented findings from preclinical research characterizing the long-term neuropathological and behavioral effects after single and repeated mild, blast-induced mTBI (rmbTBI), considered a primary risk factor for development of neurodegenerative conditions such as CTE. The pathophysiological processes linking single and multiple blast exposures to neurodegeneration, however, are not fully elucidated. These findings can be found on page S-44 of this Supplement.

Using a shock tube model in rats, exposure to a single blast resulted in (1) memory impairment that persisted for the entire 71day experimental period and (2) transient elevation of anxiety behavior that recovered to normal levels by 71 days. Proteomics analysis of brain regions mediating the above mentioned neurobehavioral functions (e.g., dorsal hippocampus, ventral hippocampus, amygdala, prefrontal cortex) from blast-exposed rats revealed region-specific changes in the levels of the select biomarkers (e.g., interferon gamma, interleukin-6), indicating that different brain regions have different vulnerability to blast exposure. Measurement of serum biomarkers suggested neuroinflammation, vascular damage, and axonal damage in these animals. These results indicate long-lasting functional and molecular changes resulting from a single blast exposure.

Inducing rmbTBI in the shock tube (five exposures, 24-h interval) resulted in more pronounced neuropathological and behavioral effects when compared with single blast exposure. Rats exposed to rmbTBI had more pronounced memory deficits (including spatial memory) and increased cell death 21 days post-injury when compared with rats exposed to a single blast. Preliminary experiments indicate that the most damaging between-exposure interval is $1 \mathrm{~h}$ in the rat model; this time frame is thought to be equivalent to several days or weeks in humans. Further defining the complex temporal relationship between human and rodent pathologies involved in the neurodegenerative processes is critical to translating pre-clinical findings to clinically relevant information useful for diagnosis of brain injury, and treatment and rehabilitation for those with such injury.

Dr. Meghan Robinson, of the VA Boston Healthcare System, presented findings from neuroimaging studies at the Translational Research Center for TBI and Stress Disorders (TRACTS) that examined the chronic effects of blast injury. Recent research suggests that acute concussion symptoms are not predictive of long-term changes to the brain. In addition, service members exposed to blast often underreport concussion, indicating that there is a population with the effects of blast injury without assessable concussion symptoms. Given these observations, investigators at TRACTS are seeking to explore variables other than concussion symptoms that could potentially characterize the chronic effects of blast injury. Two recent studies have drawn from a cohort of veterans and service members recruited by TRACTS who have completed neuropsychological testing, clinical assessment, physical assessment, and MRI scans.

One study examined alterations of functional connectivity after blast exposure. ${ }^{50}$ Subjects exposed to blast at close range $(<10 \mathrm{~m})$ exhibited decreases in functional connectivity as assessed by seedbased resting state connectively analysis of MRI scans; decreases were not observed for those exposed to longer range $(>10 \mathrm{~m})$ blasts and were independent of blast concussion. Clinical factors such as sleep, pain, or PTSD were controlled for in these comparisons. Differences in functional connectivity were not observed in subjects with concussion history.

A second study sought to determine whether blast exposure influenced age-associated degradation of white matter tissue integrity. ${ }^{51}$ Diffusion tensor imaging indicated more pronounced cross-sectional age associations with white matter integrity among subjects exposed to blast ( $<100 \mathrm{~m}$ away). Independent clinical factors, such as sleep, pain, or PTSD, could not account for this effect on white matter integrity. These observations suggest the existence of an underlying neurodegenerative process.

Taken together, these studies indicate that blast exposure may be an important consideration, independent of acute concussion symptoms, for potential chronic health effects among service members and veterans. They identify a potential clinical guideline for determining which blasts (i.e., $<10 \mathrm{~m}$ ) pose a higher relative risk to health. Complete details of Dr. Robinson's study can be found in the article "Characterization of Differences in Functional Connectivity Associated with Close-Range Blast Exposure" on page S-53 of this Supplement.

Dr. Jessica Gill, of the National Institutes of Nursing Research, reviewed the results in service members of a study of TBI biomarkers that could potentially be applied to CTE. Enhanced detection of tau and $\mathrm{A} \beta$ protein in peripheral blood has been made possible by development of a sensitive analytical technique that amplifies the signal yielded by the traditional enzyme-linked immunosorbent assay by a factor of 300 to 1000 . Analysis of peripheral blood samples was performed at Madigan Army Medical Center in service members who were deployed within the last 16 months. ${ }^{52}$ Among 70 service members who self-reported exposure to TBI during deployment, $58(82.8 \%)$ reported exposure to blast. 
The presence of tau protein in blood was associated with TBI exposure and chronic injury symptoms. The concentration of total tau protein in peripheral blood plasma was significantly higher in subjects with self- reported TBI exposure compared with nonexposed controls. Among TBI subjects, plasma concentration of tau was significantly higher in individuals reporting three or more exposures compared with those with fewer than three exposures. Total plasma levels of tau were also correlated with chronic TBI symptoms, as assessed by the Neurobehavioral Symptom Inventory.

The study's findings also indicate a potential relationship between plasma $\mathrm{A} \beta$ and blast injury. Concentration levels of $\mathrm{A} \beta$ protein were higher in subjects self-reporting that they were exposed to at least one TBI compared with controls; however, this difference was not statistically significant. Among TBI subjects exposed to blast, $\mathrm{A} \beta$ levels were significantly higher than those of nonexposed controls.

Dr. David Okonkwo, from the University of Pittsburgh Medical Center, reviewed neuroimaging research characterizing neuropathology in relation to neuropsychological outcomes associated with chronic TBI. A recent pilot study in 17 subjects with chronic TBI and four control subjects used High Definition Fiber Tracking (HDFT) to image white matter tracks, position emission tomography (PET) using Pittsburgh Compound $\mathrm{B}(\mathrm{PiB})$ to detect $\mathrm{A} \beta$ deposition, and fluorodeoxyglucose PET (FDG PET) to detect metabolic activity.

HDFT generates data specific to individual subjects by using novel quantitative metrics to circumvent mathematical limitations of voxel-based analysis; these metrics include generalized Q-space imaging algorithms followed by deterministic tractography. These quantitative metrics do not pursue group mean statistics and are specific to the individual patient. Quantitative tractography metrics can be applied in two ways: (1) examine anatomical asymmetries in white matter to identify and localize specific tracts that have been subjected to loss or abnormalities within the contralateral side of the subject's injury and also within the control subjects and (2) identify and localize abnormalities in white matter arborization, which has been observed in other studies examining subjects exposed to blast injuries.

Ongoing pilot study observations indicate a significant, ageadjusted increase of $\mathrm{A} \beta$ deposition in TBI subjects when compared with controls; this signal of increased $\mathrm{A} \beta$ was observed in brain regions vulnerable to $\mathrm{AD}$. In gray matter regions of interest in $\mathrm{AD}$, increased $\mathrm{A} \beta$ deposition was strongly associated with reduced metabolic activity, which is an expected pattern for those with dementia. In a small cohort of trauma-related dementia patients, increased $\mathrm{A} \beta$ deposition was observed in association with decreased metabolic activity, which appears to be similar to ADrelated dementia. In addition, white matter anisotropy in this cohort was associated with reduced metabolic activity and increased $\mathrm{A} \beta$ deposition.

These observations can enable testing of the hypothesis that white matter injury induces pathophysiological processes that can culminate in long-term change in humans and animal models exposed to TBI. Future research will evaluate whether white matter abnormalities are associated with functional outcomes assessed by neuropsychological tests (e.g., verbal recall, serial reaction time, processing speed index, trail making) in subjects with chronic TBI. In addition, FDG PET imaging to characterize tau protein accumulation is planned. Neuroimaging outcomes will also be correlated with cerebrospinal fluid and serum TBI biomarkers.

Dr. Dara Dickstein, from the Icahn School of Medicine at Mount Sinai, presented on behalf of Dr. Sam Gandy and described mo- lecular imaging research on TBI and CTE, including a review of case studies. Several PET ligands exist that bind with tau or amyloid protein aggregates, which are associated with TBI and CTE neuropathology. Questions remain about the binding specificity of ligands, particularly those that target tau, and whether PET binding signals are accurate reflections of neuropathology or disease states in these conditions.

The PET imaging has identified amyloid deposition after TBI. Application of the PiB radioligand indicated the presence of amyloid in acute TBI, which is retained 48 h, 54 days, and 399 days after injury. Preliminary studies in acute TBI suggest that PiB retention is observed in moderate to severe TBI with visible contusions on MRI. An ongoing PET study in boxers one month postknockout, however, did not observe amyloid retention with PiB. To date, the presence of amyloid has been associated with severe TBI, with $\mathrm{PiB}$ retention in some subjects with TBI, and neuropsychological impairment. ${ }^{53}$

Investigators have sought to characterize tau after TBI with PET imaging. ${ }^{54-56}$ These studies indicate the presence of p-tau in varying brain distribution patterns, bringing to light questions about the binding specificity of tau ligands and the potential contribution of age-related tauopathy. Studies have indicated that age-related tauopathy is predominately localized in the hippocampus and entorhinal cortex as opposed to a case with severe AD with p-tau staining extending into the temporal neocortex. ${ }^{57}$ To establish that these ligands can be used to study CTE, it is important to characterize the distribution patterns of $\mathrm{p}$-tau in agerelated tauopathy and AD.

Recent case reports in two subjects, aged 68 and 70 years, with TBI history and memory-related neurocognitive problems yielded mixed results: One was tau positive and amyloid negative, while the other was tau negative and amyloid positive. These observations have led investigators to ask whether CTE can be distinguished from AD using PET imaging and cognitive assessment. An ongoing study to address this question is seeking to image amyloid (using the $\left[{ }^{18} \mathrm{~F}\right]$-florbetapir ligand) and tau (using the $\left[{ }^{18} \mathrm{~F}\right]-\mathrm{T} 807$ ligand) in conjunction with neuropsychological assessment in subjects with a history of concussion; controls have been the most difficult study group to recruit.

An additional case report yielded neuroimaging findings that appear to be associated with behavioral symptoms. The subject was a 39-year-old retired professional football player with multiple concussions and severe functional impairment, including sleep disturbances, headaches, short-term memory loss, as well as impulsive and aggressive behaviors. The subject had kept meticulous neuroimaging, neuropsychological assessment, and concussion history records, enabling longitudinal evaluation.

Neurocognitive assessments indicated a five-year decline in several functional areas, including executive function, verbal reasoning, motor skills, narrative memory, and word retrieval. The MRI records showed diffuse cortical thinning in several brain areas; this was most pronounced in the left frontal and temporal areas, which correlate with areas of the brain governing the neuropsychological functions that declined in this subject. Subcortical volume decrease was greatest in the globus pallidus. The $\left[{ }^{18} \mathrm{~F}\right]-$ florbetapir PET imaging for amyloid deposition was negative in this subject, but binding of $\left[{ }^{18} \mathrm{~F}\right]-\mathrm{T} 807$ ligand indicated the presence of tau aggregation. The disparate observations of tau aggregation in these case studies and other findings indicate a need for scientific progress and consensus among experts on the interpretation of tau signal in PET imaging. 


\section{Treatment strategies}

Although the neuropathological and clinical features of CTE have not been definitively established, investigators have made advances in exploring potential treatment strategies for chronic neurodegeneration, including CTE.

Dr. Kun Ping Lu, of the Beth Israel Deaconess Medical Center at Harvard Medical School, reviewed research about the role of tauopathy in TBI, CTE, and AD and the development of novel antibodies that specifically recognize the early driver of neurodegeneration, cis p-tau. Although TBI is a potential risk factor for both CTE and AD, and tauopathy is a primary neuropathological characteristic of $\mathrm{CTE}$ and $\mathrm{AD}$, the pathogenic processes linking TBI exposure to the development of tauopathy remain largely unknown.

In $\mathrm{AD}$ studies, the $c i$ is isomer of p-tau is predominantly localized to axons and has been implicated in the pathogenesis of $\mathrm{AD}$ in rodents and human brain tissue. ${ }^{58}$ Unlike trans p-tau, the $c$ is isomer cannot promote microtubule assembly, is more resistant to dephosphorylation and degradation, and is more prone to aggregation. When cis p-tau is converted to the trans conformation, through Pin1, a peptidyl-prolyl cis-trans isomerase, the progression of tauopathy in $\mathrm{AD}$ is inhibited, suggesting that the cis isomer of p-tau may be a promising biomarker and therapeutic target of AD.

Recent neuropathological studies indicate the presence of cis p-tau in human brains with CTE after TBI. The cis isoform appears at early time points in mouse brain tissue after exposure to blastand impact-induced TBI. ${ }^{61}$ Forty-eight hours after blast, cis p-tau is localized in neurons of the cortex in the damaged side of the brain and spreads to hippocampal neurons in the ipsilateral hemisphere by two weeks and then to the cortex in the contralateral hemisphere by three months. Follow-on experiments in cell culture found that repeated TBI brain lysates potently induce cell death in $22 \%$ of neurons by apoptosis; when cis p-tau is depleted from the TBI brain lysates by cis p-tau antibody, neuron apoptosis is inhibited. In addition, cis p-tau antibody blocks the ability of neuronal stress, such as hypoxia, to induce cis p-tau and apoptosis in cultured neurons.

The cis p-tau-induced pathogenic process termed "cistauosis" is thought to mediate disease progression of tauopathy through disruption of axonal microtubule networks and mitochondrial transport, ultimately leading to apoptosis; these events are blocked by cis p-tau antibody in neuron cultures and rodents. ${ }^{59}$ Blockade of cistauosis in mice exposed to TBI through administration of cis $\mathrm{p}$ tau antibody prevents tauopathy; prevents neuron loss and brain atrophy; and restores TBI-related functional outcomes, including risk-taking behavior, urinary dysfunction, and sensorimotor imbalance. Cistauosis is thought to represent a pathogenic mechanism linking TBI to AD and CTE; cis p-tau antibody may be used to target this mechanism for the development of early diagnosis, prevention, and therapeutic strategies for these conditions.

\section{Conclusion}

There are many efforts under way currently to mitigate threats to brain health for service members. Throughout their military career, service members may face many types of brain health threats, including blast injury and head trauma; specific threats are determined by adversary, conflict environment, and operational realities. The DoD seeks to mitigate the risks service members face by enhancing warfighter health, fitness, protection, and resilience, as well as improving healthcare delivery and those systems that support recovery, rehabilitation, and reintegration. For example, today's service members are better prepared to deploy and fight in high-altitude regions because of research determining the mitigating factors for pulmonary and cerebral effects of altitude sickness.

Responding to service member health needs is challenging because of changing threats and the fact that responsible utilization of mitigation strategies requires testing to ensure quality, safety, and effectiveness. The DoD is working to meet these challenges, with both material products and the knowledge needed to prevent, mitigate, and reduce health risks in a tactical environment.

Our understanding of the long-term effects of TBI, however, is an endeavor still in its infancy. More research is needed to establish the existence of links between the long-term effects of single or multiple concussions and CTE. The presentations summarized in this article provided the background and common understanding for an expert panel discussion on the possible relationship between TBI and CTE. Findings and recommendations resulting from this meeting will help guide future scientific research and the development of prevention, assessment, and treatment strategies.

\section{Author Disclosure Statement}

No competing financial interests exist.

\section{References}

1. Glenn, J. (2015). Keynote address. Presented at the 2015 International State-of-the-Science meeting, McLean, VA.

2. Hinds, S. (2015). Prevalence of TBI and chronic effects of blastrelated trauma in the military. Presented at the 2015 International State-of-the-Science meeting, McLean, VA.

3. Da Silva, U.U. (2015). Annual blast overpressure exposure of mortar and artillery men. Presented at the 2015 International State-of-theScience meeting, McLean, VA.

4. Lattimore, T. and Fudge, E. (2015). Future policy considerations for managing chronic effects of TBI in service members. Presented at the 2015 International State-of-the-Science meeting, McLean, VA.

5. Hoffman, S. (2015). VA perspective on current state of the science and policy considerations: Past, present, and future. Presented at the 2015 International State-of-the-Science meeting, McLean, VA.

6. Bellgowan, P. (2015). NIH-Public Health perspective on the relationship between head trauma and CTE. Presented at the 2015 International State-of-the-Science meeting, McLean, VA.

7. Broglio, S.P. (2015). NCAA perspective on the relationship between head trauma and CTE. Presented at the 2015 International State-ofthe-Science meeting, McLean, VA.

8. Cameron, K.L., Marshall, S.W., Sturdivant, R.X., and Lincoln, A.E. (2012). Trends in the incidence of physician-diagnosed mild traumatic brain injury among active duty U.S. military personnel between 1997 and 2007. J. Neurotrauma 29, 1313-1321.

9. Guskiewicz, K.M., Marshall, S.W., Bailes, J., McCrea, M., Cantu, R.C., Randolph, C., and Jordan, B.D. (2005). Association between recurrent concussion and late-life cognitive impairment in retired professional football players. Neurosurgery 57, 719-726.

10. Broglio, S.P., Ferrara, M.S., Piland, S.G., Anderson, R.B., and Collie, A. (2006). Concussion history is not a predictor of computerised neurocognitive performance. Br. J. Sports Med. 40, 802-805.

11. Broglio, S.P., Pontifex, M.B., O'Connor, P., and Hillman, C.H. (2009). The persistent effects of concussion on neuroelectric indices of attention. J. Neurotrauma 26, 1463-1470.

12. Martini, D.N., Sabin, M.J., DePesa, S.A., Leal, E.W., Negrete, T.N., Sosnoff, J.J., and Broglio, S.P. (2011). The chronic effects of concussion on gait. Arch. Phys. Med. Rehabil. 92, 585-589.

13. Sosnoff, J.J., Broglio, S.P., Shin, S., and Ferrara, M.S. (2011). Previous mild traumatic brain injury and postural-control dynamics. J. Athl. Train. 46, 85-91.

14. Broglio, S.P., Eckner, J.T., Paulson, H.L., and Kutcher, J.S. (2012). Cognitive decline and aging: the role of concussive and subconcussive impacts. Exerc. Sport Sci. Rev. 40, 138-144.

15. Maroon, J. (2015). Chronic traumatic encephalopathy: Contact sports and blast injuries. Presented at the 2015 International State-of-theScience meeting, McLean, VA. 
16. Giza, C.C., and Hovda, D.A. (2001). The neurometabolic cascade of concussion. J. Athl. Train. 36, 228-235.

17. Giza, C.C. and Hovda, D.A. (2014). The new neurometabolic cascade of concussion. Neurosurgery 75, Suppl 4, S24-S33.

18. Blaylock, R.L. and Maroon, J. (2011). Immunoexcitotoxicity as a central mechanism in chronic traumatic encephalopathy-A unifying hypothesis. Surg. Neurol. Int. 2, 107.

19. Bloomfield, P.S., Selvaraj, S., Veronese, M., Rizzo, G., Bertoldo, A., Owen, D.R., Bloomfield, M.A., Bonoldi, I., Kalk, N., Turkheimer, F., McGuire, P., de Paola, V., and Howes, O.D. (2016). Microglial activity in people at ultra high risk of psychosis and in schizophrenia: An [(11)C]PBR28 PET brain imaging study. Am. J. Psychiatry 173, 44-52.

20. von Känel, R., Hepp, U., Kraemer, B., Traber, R., Keel, M., Mica, L., and Schnyder, U. (2007). Evidence for low-grade systemic proinflammatory activity in patients with posttraumatic stress disorder. J. Psychiatr. Res. 41, 744-752.

21. Lu, J., Ng, K.C., Ling, G., Wu, J., Poon, D.J., Kan, E.M., Tan, M.H., Wu, Y.J., Li, P., Moochhala, S., Yap, E., Lee, L.K., Teo, M., Yeh, I.B., Sergio, D.M., Chua, F., Kumar, S.D., and Ling, E.A. (2012) Effect of blast exposure on the brain structure and cognition in Macaca fascicularis. J. Neurotrauma 29, 1434-1454.

22. Bauman, R.A., Ling, G., Tong, L., Januszkiewicz, A., Agoston, D., Delanerolle, N., Kim, Y., Ritzel, D., Bell, R., Ecklund, J., Armonda, R., Bandak, F., and Parks, S. (2009). An introductory characterization of a combat-casualty-care relevant swine model of closed head injury resulting from exposure to explosive blast. J. Neurotrauma 26, 841-860.

23. Long, J.B., Bentley, T.L., Wessner, K.A., Cerone, C., Sweeney, S., and Bauman, R.A. (2009). Blast overpressure in rats: recreating a battlefield injury in the laboratory. J. Neurotrauma 26, 827-840.

24. Risling, M., Plantman, S., Angeria, M., Rostami, E., Bellander, B.M., Kirkegaard, M., Arborelius, U., and Davidsson, J. (2011). Mechanisms of blast induced brain injuries, experimental studies in rats. NeuroImage 54, Suppl 1, S89-S97.

25. Ryu, J., Horkayne-Szakaly, I., Xu, L., Pletnikova, O., Leri, F., Eberhart, C., Troncoso, J.C., and Koliatsos, V.E. (2014). The problem of axonal injury in the brains of veterans with histories of blast exposure. Acta Neuropathol. Commun. 2, 153.

26. Säljö, A., Bao, F., Hamberger, A., Haglid, K.G., and Hansson, H.A. (2001). Exposure to short-lasting impulse noise causes microglial and astroglial cell activation in the adult rat brain. Pathophysiology 8 , 105-111.

27. McKee, A.C., Stern, R.A., Nowinski, C.J., Stein, T.D., Alvarez, V.E., Daneshvar, D.H., Lee, H.S., Wojtowicz, S.M., Hall, G., Baugh, C.M., Riley, D.O., Kubilus, C.A., Cormier, K.A., Jacobs, M.A., Martin, B.R., Abraham, C.R., Ikezu, T., Reichard, R.R., Wolozin, B.L., Budson, A.E., Goldstein, L.E., Kowall, N.W., and Cantu, R.C. (2013). The spectrum of disease in chronic traumatic encephalopathy. Brain 136, 43-64.

28. Stein, T.D., Montenigro, P.H., Alvarez, V.E., Xia, W., Crary, J.F., Tripodis, Y., Daneshvar, D.H., Mez, J., Solomon, T., Meng, G., Kubilus, C.A., Cormier, K.A., Meng, S., Babcock, K., Kiernan, P., Murphy, L., Nowinski, C.J., Martin, B., Dixon, D., Stern, R.A., Cantu, R.C., Kowall, N.W., and McKee, A.C. (2015). Beta-amyloid deposition in chronic traumatic encephalopathy. Acta Neuropathol. 130, 21-34.

29. McKee, A.C., Alvarez, V., Bieniek, K., Cairns, N., Crary, J., DamsO'Connor, K., Folkerth, R., Keene, C.D., Litvan, I., Montine, T., Montenigro, P., Perl, D., Stein, T., Stewart, W., Tripodis, Y., Vonsattel, J.P., Gordon, W., and Dickson, D. (2015). Preliminary results of the NINDS/NIBIB Consensus Meeting to evaluate pathological criteria for the diagnosis of CTE (P2.178). Neurology 84, P2.178.

30. Stern, R.A., Daneshvar, D.H., Baugh, C.M., Seichepine, D.R., Montenigro, P.H., Riley, D.O., Fritts, N.G., Stamm, J.M., Robbins, C.A., McHale, L., Simkin, I., Stein, T.D., Alvarez, V.E., Goldstein, L.E., Budson, A.E., Kowall, N.W., Nowinski, C.J., Cantu, R.C., and McKee, A.C. (2013). Clinical presentation of chronic traumatic encephalopathy. Neurology 81, 1122-1129.

31. Montenigro, P.H., Baugh, C.M., Daneshvar, D.H., Mez, J., Budson, A.E., Au, R., Katz, D.I., Cantu, R.C., and Stern, R.A. (2014). Clinical subtypes of chronic traumatic encephalopathy: literature review and proposed research diagnostic criteria for traumatic encephalopathy syndrome. Alzheimers Res. Ther. 6, 68.
32. Montenigro, P.H., Bernick, C., and Cantu, R.C. (2015). Clinical features of repetitive traumatic brain injury and chronic traumatic encephalopathy. Brain Pathol. 25, 304-317.

33. Mez, J., Solomon, T.M., Daneshvar, D.H., Murphy, L., Kiernan, P.T., Montenigro, P.H., Kriegel, J., Abdolmohammadi, B., Fry, B., Babcock, K.J., Adams, J.W., Bourlas, A.P., Papadopoulos, Z., McHale, L., Ardaugh, B.M., Martin, B.R., Dixon, D., Nowinski, C.J., Chaisson, C., Alvarez, V.E., Tripodis, Y., Stein, T.D., Goldstein, L.E., Katz, D.I., Kowall, N.W., Cantu, R.C., Stern, R.A., and McKee, A.C. (2015). Assessing clinicopathological correlation in chronic traumatic encephalopathy: rationale and methods for the UNITE study. Alzheimers Res. Ther. 7, 62 .

34. Löwenhielm, P. (1975). Mathematical simulation of gliding contusions. J. Biomech. 8, 351-356.

35. Ahlers, S.T., Vasserman-Stokes, E., Shaughness, M.C., Hall, A.A., Shear, D.A., Chavko, M., McCarron, R.M., and Stone, J.R. (2012). Assessment of the effects of acute and repeated exposure to blast overpressure in rodents: toward a greater understanding of blast and the potential ramifications for injury in humans exposed to blast. Front. Neurol. 3, 32.

36. Haghighi, F., Ge, Y., Chen, S., Xin, Y., Umali, M.U., De Gasperi, R., Gama Sosa, M.A., Ahlers, S.T., and Elder, G.A. (2015). Neuronal DNA methylation profiling of blast-related traumatic brain injury. J. Neurotrauma 32, 1200-1209.

37. DeKosky, S.T., Abrahamson, E.E., Ciallella, J.R., Paljug, W.R., Wisniewski, S.R., Clark, R.S.B., and Ikonomovic, M.D. (2007). Association of increased cortical soluble abeta42 levels with diffuse plaques after severe brain injury in humans. Arch. Neurol. 64, 541-544.

38. Loane, D.J., Pocivavsek, A., Moussa, C.E., Thompson, R., Matsuoka, Y., Faden, A.I., Rebeck, G.W., and Burns, M.P. (2009). Amyloid precursor protein secretases as therapeutic targets for traumatic brain injury. Nat. Med. 15, 377-379.

39. De Gasperi, R., Gama Sosa, M.A., Kim, S.H., Steele, J.W., Shaughness, M.C., Maudlin-Jeronimo, E., Hall, A.A., Dekosky, S.T., McCarron, R.M., Nambiar, M.P., Gandy, S., Ahlers, S.T., and Elder, G.A. (2012). Acute blast injury reduces brain abeta in two rodent species. Front. Neurol. 3, 177.

40. Elder, G.A., Gama Sosa, M.A., De Gasperi, R., Stone, J.R., Dickstein, D.L., Haghighi, F., Hof, P.R., and Ahlers, S.T. (2015). Vascular and inflammatory factors in the pathophysiology of blast-induced brain injury. Front. Neurol. 6, 48.

41. Ross, B.D., Ernst, T., Kreis, R., Haseler, L.J., Bayer, S., Danielsen, E. Blüml, S., Shonk, T., Mandigo, J.C., Caton, W., Clark, C., Jensen, S.W., Lehman, N.L., Arcinue, E., Pudenz, R., and Shelden, C.H. (1998). 1H MRS in acute traumatic brain injury. J. Magn. Reson. Imaging 8, 829-840.

42. Shutter, L., Tong, K.A., and Holshouser, B.A. (2004). Proton MRS in acute traumatic brain injury: role for glutamate/glutamine and choline for outcome prediction. J. Neurotrauma 21, 1693-1705.

43. Vagnozzi, R., Signoretti, S., Tavazzi, B., Floris, R., Ludovici, A., Marziali, S., Tarascio, G., Amorini, A.M., Di Pietro, V., Delfini, R. and Lazzarino, G. (2008). Temporal window of metabolic brain vulnerability to concussion: a pilot $1 \mathrm{H}$-magnetic resonance spectroscopic study in concussed athletes-part III. Neurosurgery 62, 1286-1295.

44. Lin, A., Liao, H., Merugumala, S., Stern, R., and Ross, B. (2014) Regional Metabolite Profiles in Chronic Sports-Related Concussion. Presented at the 10th World Congress on Brain Injury, San Francisco, CA.

45. Koerte, I.K., Lin, A.P., Muehlmann, M., Merugumala, S., Liao, H., Starr, T., Kaufmann, D., Mayinger, M., Steffinger, D., Fisch, B., Karch, S., Heinen, F., Ertl-Wagner, B., Reiser, M., Stern, R.A., Zafonte, R., and Shenton, M.E. (2015). Altered neurochemistry in former professional soccer players without a history of concussion. J. Neurotrauma 32, 1287-1293.

46. Corsellis, J.N., Bruton, C.J., and Freeman-Browne, D. (1973). The aftermath of boxing. Psychol. Med. 3, 270-303.

47. Hoozemans, J.J., van Haastert, E.S., Nijholt, D.A., Rozemuller, A.J., Eikelenboom, P., and Scheper, W. (2009). The unfolded protein response is activated in pretangle neurons in Alzheimer's disease hippocampus. Am. J. Pathol. 174, 1241-1251.

48. Ho, Y.S., Yang, X., Lau, J.C.., Hung, C.H., Wuwongse, S., Zhang, Q., Wang, J., Baum, L., So, K.F., and Chang, R.C. (2012). Endoplasmic reticulum stress induces tau pathology and forms a vicious cycle: 
implication in Alzheimer's disease pathogenesis. J. Alzheimers Dis. 28, 839-854.

49. Nijholt, D.A., van Haastert, E.S., Rozemuller, A.J., Scheper, W., and Hoozemans, J.J. (2012). The unfolded protein response is associated with early tau pathology in the hippocampus of tauopathies. J. Pathol. 226, 693-702.

50. Robinson, M.E., Lindemer, E.R., Fonda, J.R., Milberg, W.P., McGlinchey, R.E., and Salat, D.H. (2015). Close-range blast exposure is associated with altered functional connectivity in Veterans independent of concussion symptoms at time of exposure. Hum. Brain Mapp. 36, 911-922.

51. Trotter, B.B., Robinson, M.E., Milberg, W.P., McGlinchey, R.E., and Salat, D.H. (2015). Military blast exposure, ageing and white matter integrity. Brain 138, 2278-2292.

52. Olivera, A., Lejbman, N., Jeromin, A., French, L.M., Kim, H.S., Cashion, A., Mysliwiec, V., Diaz-Arrastia, R., and Gill, J. (2015). Peripheral total tau in military personnel who sustain traumatic brain injuries during deployment. JAMA Neurol. 72, 1109-1116.

53. Kawai, N., Kawanishi, M., Kudomi, N., Maeda, Y., Yamamoto, Y., Nishiyama, Y., and Tamiya, T. (2013). Detection of brain amyloid $\beta$ deposition in patients with neuropsychological impairment after traumatic brain injury: PET evaluation using Pittsburgh Compound-B. Brain Inj. 27, 1026-1031.

54. Barrio, J., Small, G.W., Wong, K.P., Huang, S.C., Liu, J., Merrill, D.A., Giza, C.C., Fitzsimmons, R.P., Omalu, B., Bailes, J., and Kepe, V. (2015). In vivo characterization of chronic traumatic encephalopathy using [F-18]FDDNP PET brain imaging. Proc. Natl. Acad. Sci. U. S. A. 112, E2039-E2047.

55. Chien, D.T., Bahri, S., Szardenings, A.K., Walsh, J.C., Mu, F., Su, M.Y., Shankle, W.R., Elizarov, A., and Kolb, H.C. (2013). Early clinical PET imaging results with the novel PHF-tau radioligand [F18]-T807. J. Alzheimers Dis. JAD 34, 457-468.

56. Mitsis, E.M., Riggio, S., Kostakoglu, L., Dickstein, D.L., Machac, J., Delman, B., Goldstein, M., Jennings, D., D’Antonio, E., Martin, J., Naidich, T.P., Aloysi, A., Fernandez, C., Seibyl, J., DeKosky, S.T., Elder, G.A., Marek, K., Gordon, W., Hof, P.R., Sano, M., and Gandy,
S. (2014). Tauopathy PET and amyloid PET in the diagnosis of chronic traumatic encephalopathies: studies of a retired NFL player and of a man with FTD and a severe head injury. Transl. Psychiatry 4, e441.

57. Crary, J.F., Trojanowski, J.Q., Schneider, J.A., Abisambra, J.F., Abner, E.L., Alafuzoff, I., Arnold, S.E., Attems, J., Beach, T.G., Bigio, E.H., Cairns, N.J., Dickson, D.W., Gearing, M., Grinberg, L.T., Hof, P.R., Hyman, B.T., Jellinger, K., Jicha, G.A., Kovacs, G.G., Knopman, D.S., Kofler, J., Kukull, W.A., Mackenzie, I.R., Masliah, E., McKee, A., Montine, T.J., Murray, M.E., Neltner, J.H., Santa-Maria, I., Seeley, W.W., Serrano-Pozo, A., Shelanski, M.L., Stein, T., Takao, M., Thal, D.R., Toledo, J.B., Troncoso, J.C., Vonsattel, J.P., White, C.L., Wisniewski, T., Woltjer, R.L., Yamada, M., and Nelson, P.T. (2014). Primary age-related tauopathy (PART): a common pathology associated with human aging. Acta Neuropathol. 128, 755-766.

58. Nakamura, K., Greenwood, A., Binder, L., Bigio, E.H., Denial, S., Nicholson, L., Zhou, X.Z., and Lu, K.P. (2012). Proline isomerspecific antibodies reveal the early pathogenic tau conformation in Alzheimer's disease. Cell 149, 232-244.

59. Kondo, A., Shahpasand, K., Mannix, R., Qiu, J., Moncaster, J., Chen, C.H., Yao, Y., Lin, Y.M., Driver, J.A., Sun, Y., Wei, S., Luo, M.L., Albayram, O., Huang, P., Rotenberg, A., Ryo, A., Goldstein, L.E., Pascual-Leone, A., McKee, A.C., Meehan, W., Zhou, X.Z., and Lu, K.P. (2015). Antibody against early driver of neurodegeneration cis Ptau blocks brain injury and tauopathy. Nature 523, 431-436.

Address correspondence to: Raj K. Gupta, PhD Department of Defense Blast Injury Research Program Coordinating Office

United States Army Medical Research and Materiel Command 504 Scott Street

Fort Detrick, MD 21702

E-mail: raj.k.gupta.civ@mail.mil 